\title{
Revisión de tecnologías habilitadoras para el control biológico y de plagas en el sector hortofrutícola
}

\author{
Cristian Martín, Luis Llopis, Bartolomé Rubio, Manuel Díaz \\ ITIS Software, Universidad de Málaga, Málaga, España, (e-mail: cmf@lcc.uma.es) \\ Fernando Díaz \\ Software for Critical Systems SL, Arquitecto Francisco Peñalosa, 18, 29010, Málaga, España
}

\begin{abstract}
Resumen
La monitorización continua tanto de plagas que afectan a los cultivos hortofrutícolas en agricultura intensiva de invernadero, como de la lucha biológica, utilizada en este tipo de cultivos para combatir las plagas de una forma sostenible con el medio ambiente son esenciales en este sector. Actualmente, en la mayoría de los casos este control se realiza de forma manual (no siempre sin dificultad), lo que requiere a los agricultores de una supervisión continua para poder detectar, en el menor tiempo posible, cualquier brote que afecte a sus cultivos. En este trabajo se presenta una revisión de tecnologías habilitadoras para permitir una monitorización continua, sostenible y de forma autónoma en este sector estratégico en España. Estas tecnologías se integran en una propuesta de sistema de ayuda a la decisión para el control de plagas y control biológico.
\end{abstract}

Palabras clave: Control biológico; control de plagas; sector hortofruticola; arquitecturas multinivel; vehículo de inspección autónomo; inteligencia artificial

\section{Introducción}

España es una de las mayores regiones agrícolas de Europa, y en concreto su provincia de Almería, también conocida como la "Huerta de Europa", produce y exporta un amplio abanico de productos hortofrutícolas gracias a la explotación de la agricultura intensiva en su "Mar de Plástico". Esta actividad económica ha sido el principal motor económico de esta región desde los años 60 hasta hoy en día, siendo la región con la mayor concentración de invernadero de Europa. El camino hacia la producción sostenible, el posicionamiento de los productos hortofrutícolas andaluces en mercados internacionales y la mejora en la gestión de plagas, llevaron a la incorporación y la obligatoriedad de la Gestión Integrada de Plagas (GIP) [4] y la adopción de mecanismos de sostenibilidad como el control biológico de plagas.

El control biológico de plagas permite emplear elementos y organismos biológicos respetuosos con el medio ambiente y con garantías sanitarias para el control de plagas, lo que se traduce en una disminución del uso de productos fitosanitarios $\mathrm{y}$, por tanto, en una mejora en la seguridad alimentaria y la calidad resultante de los productos hortofrutícolas, libres de residuos. Ejemplos de control biológico de plagas son el Orius laevigatus y Amblyseius swirskii, especies que permiten luchar y combatir contra el trips (Frankliniella occidentalis) y la mosca blanca (Bemisia tabaci) que hacen un especial daño a explotaciones hortofrutícolas como la del pimiento. Al ser depredadores naturales de trips y mosca blanca, y dado el especial daño que hacen estas plagas a los cultivos de pimiento, su presencia en invernaderos es crítica para el control de estas plagas de manera sostenible. A pesar de los beneficios que aportan este tipo de depredadores para el control biológico de plagas, su detección no siempre es sencilla, ya que en muchos casos estos depredadores se desplazan en el envés de las hojas de las plantas y poseen de un tamaño muy reducido (ver Figura 1). Por tanto, resulta a veces muy tedioso, lo que requiere de pasadas manuales y con una lupa, tener una visión clara del estado de los invernaderos con respecto al control biológico. Además, actualmente el tejido agrario carece de una solución autónoma que lo permita.

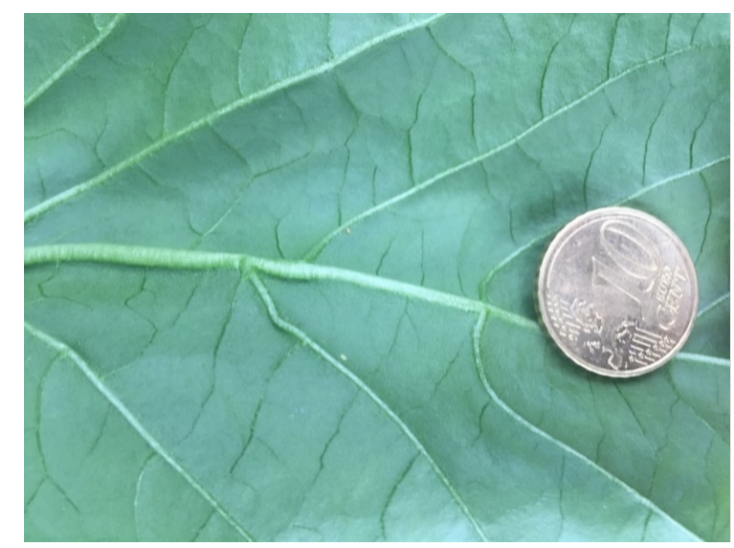

Figura 1: Especies Amblyseius swirskii de control biológico presentes el envés de una hoja de pimiento

Cuando una plaga se expande y daña seriamente 
a los cultivos, es fácilmente detectable a simple vista ya que las hojas pueden cambiar de color y la planta detiene drásticamente su crecimiento. Sin embargo, cuando éstas se desplazan a lo largo de las plantaciones, pueden ser difícil de localizar, ya que pueden poseer un carácter diminuto al igual que las especies depredadoras. Por otro lado, la aplicación de las especies de control biológico es llevada a cabo en diferentes etapas de las plantaciones hortofrutícolas, siguiendo normalmente un proceso manual y seguido por un patrón preestablecido, como, por ejemplo, depositando una solución biológica cada 5-6 plantas (ver Figura 2 ). Sin embargo, al igual que las plagas, la presencia de estos organismos puede variar a lo largo del ciclo de vida de los cultivos hortofrutícolas, por lo que una monitorización continua del cultivo es actualmente requerida tanto por los propios agricultores como por los técnicos agrícolas. Por otro lado, las características de las plantaciones y su biodiversidad presente pueden dar lugar a otro tipo de disposición de las soluciones de control biológico, que puede tener una repercusión económica positiva para los agricultores, y para la que la monitorización continua es necesaria para asegurar su habitabilidad. El exceso de control biológico puede dar lugar también a que los depredadores naturales empiecen a consumir recursos de las plantas para su alimentación y mermen su rendimiento, por lo que un perfecto equilibrio entre plagas y control bilógico es requerido para asegurar la biodiversidad de especies y un óptimo ambiente para los cultivos hortofrutícolas.

En el presente trabajo se presenta una revisión de tecnologías habilitadoras para mejorar la sostenibilidad en el sector hortofrutícola, en concreto, para ofrecer un sistema de monitorización autónonomo del control biológico y de plagas en cultivos de invernadero, que son elementos clave para el desarrollo de sus productos. Estas tecnologías se dividen en tres principales líneas: 1) arquitecturas multinivel para optimización de latencia y computación de soluciones IoT (Sección 2); 2) vehículos de inspección autónomos (Sección 3); y 3) técnicas de visión por computador e inteligencia artificial (Sección 4). Por último, en la Sección 5 se presenta una propuesta de sinergia de estas tecnologías y como pueden integrarse para contribuir con el objetivo propuesto.

\section{Arquitecturas multinivel para optimización de latencia y computación de soluciones IoT}

El Internet de las cosas (IoT) [5] es una tecnología que involucra un conjunto de objetos conectados entre sí a través de Internet y que se caracteriza

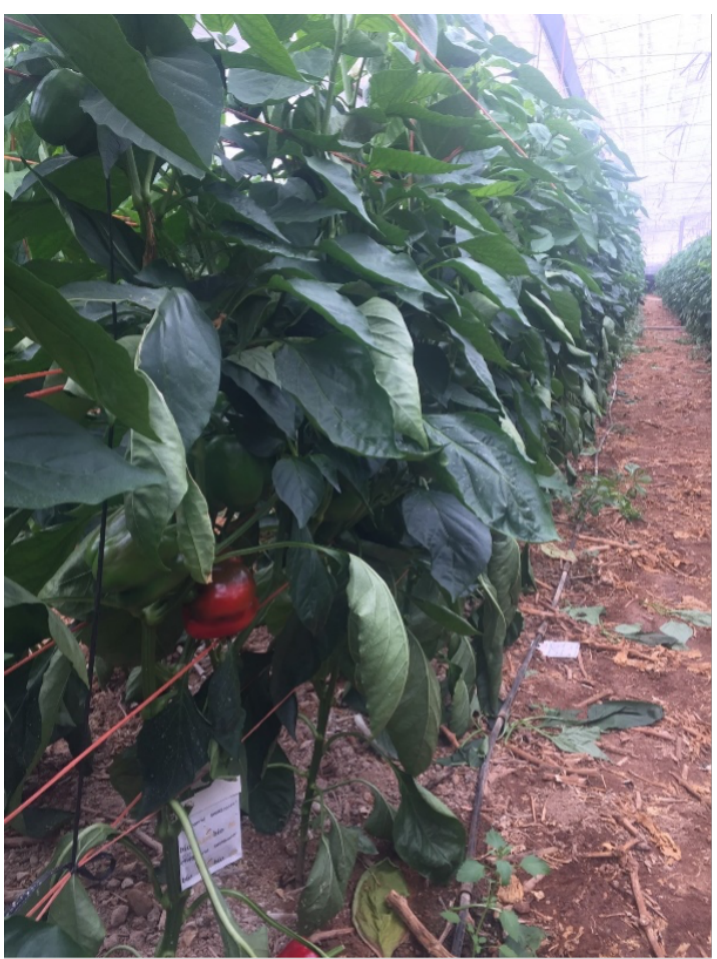

Figura 2: Solución depositada de Amblyseius swirskii para el control biológico en cultivo de pimiento

por ser una tecnología ubicua que está presente en un gran número de áreas, como puede ser la monitorización de infraestructuras críticas, smart home, smart city, sistemas de monitorización o sistemas asistidos para el cuidado de la salud. El IoT está cada vez más presente en nuestro día a día, cubriendo un gran abanico de posibilidades con el fin de optimizar los procesos y problemas a los que se enfrenta la sociedad. Sin embargo, los dispositivos involucrados en el IoT se corresponden normalmente con dispositivos embebidos con limitaciones de almacenamiento y procesamiento, así como restricciones de memoria y potencia. Además, el número de objetos o dispositivos conectados a Internet contiene grandes previsiones de crecimiento para los próximos años, con unas expectativas de 500 mil millones de objetos conectados para 2030 según CISCO.

Por lo tanto, para dar cabida a despliegues del IoT, además de suplir las limitaciones que existen, es necesario involucrar nuevos sistemas y paradigmas que faciliten la adopción de este campo. Diferentes integraciones han sido investigadas para suplir las limitaciones del IoT [5]. Entre ellas, cabe destacar a cloud computing (computación en la nube), también conocido como el cloud de las cosas, que ha intentado solventar las limitaciones del IoT. Cloud computing permite un acceso bajo demanda a un conjunto configurable 


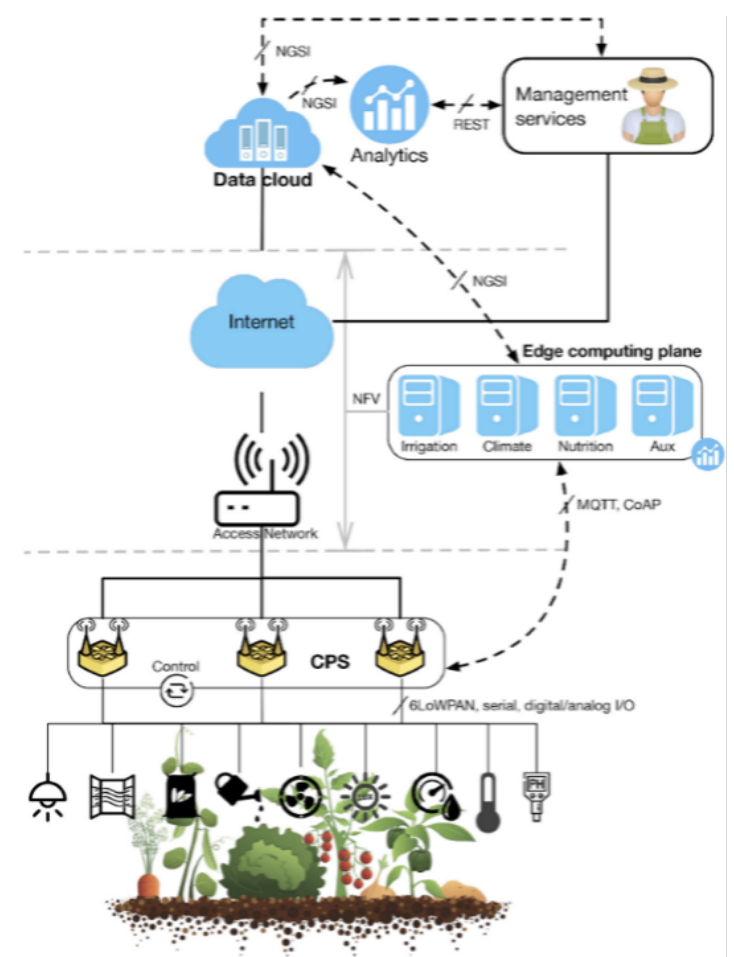

Figura 3: Visión general de la arquitectura utilizada para agricultura de precisión. Fuente: [20]

de recursos, proveyendo capacidades ilimitadas en términos de almacenamiento y procesamiento, las principales limitaciones en el IoT. Aunque la integración con cloud computing provee al IoT de las capacidades mencionadas, las necesidades actuales de extraer conocimiento de información en tiempo real implican pensar más allá. Recientes paradigmas como fog y edge computing intentan abordar este último problema, moviendo el procesamiento lo más cerca posible a dónde es generado, reduciendo por tanto la latencia y el ancho de banda en las comunicaciones entre el IoT y el cloud. En el contexto de la agricultura, este tipo de paradigmas ha sido aplicado en múltiples procesos en los que cabe destacar la agricultura de precisión y el estado de plantaciones [8]; marcos de trabajo para facilitar a los usuarios la simulación de escenarios agrícolas personalizados, específicamente para identificar la ubicación sensores, el área de cobertura y la implementación de la línea de visión [2]; y la gestión eficiente del cultivo en invernaderos [14]. También han sido aplicados a técnicas de visión por computador para mejorar los tiempos de latencia y cómputo [7]. La Figura 3 muestra un ejemplo de la aplicación de edge computing para optimizar el análisis de información en la agricultura de precisión.

Existe actualmente una tendencia para desplegar los algoritmos de visión por computador en el ciclo de cómputo que va desde el IoT hasta el cloud, incluyendo paradigmas como el edge y fog computing, para diversos fines, como el soporte para acciones críticas y la reducción del ancho de banda a partir del particionamiento, a veces dinámico [9] de las capas involucradas. Se ha demostrado que tener múltiples capas de estos algoritmos puede aumentar la solidez del reconocimiento de patrones, pero también reducir la latencia de ejecución si estas están distribuidas en diferentes niveles y pueden tener salidas de predicción intermedias [17]. Por esta razón, las capas de estos algoritmos se están distribuyendo en arquitecturas multicapa tales como edge y fog en el continuo IoT-cloud. La monitorización, la administración y la implementación adaptable de estos algoritmos distribuidos están actualmente limitadas, lo que puede dificultar el desarrollo de estas aplicaciones. Nuevas arquitecturas y capas de comunicación flexibles son necesarias en este campo.

\section{Vehículos de inspección autónomos}

Un vehículo de inspección puede ofrecer una solución no intrusiva para evaluar el estado de los cultivos con respecto a las plagas existentes y el control biológico. Este debe realizar sus tareas de forma completamente autónoma, siendo capaz de llevar a cabo la inspección sin intervención humana. Para ello, el vehículo debe resolver una serie de tareas por si solo que son habituales en la robótica móvil, como la localización, planificación y control de movimientos. Estas cuestiones, aunque habituales en el campo de la robótica móvil, son complejas en el sector hortofrutícola, debido a las características del entorno en el que se debe desenvolver su trabajo: un entorno de interior no estructurado. El hecho de tratarse de un entorno interior sin una estructura geométrica regular, como el interior de cualquier edificio o construcción, y un terreno inestable, conlleva concentrar las características más desfavorables para un sistema autónomo.

La principal desventaja de un entorno interior frente a uno exterior es la imposibilidad de utilizar el sistema GPS para determinar la localización de un vehículo en la superficie terrestre [19]. Contar con la localización proporcionada por un GPS alivia enormemente el problema de localización de un vehículo. Por tanto, en un entorno interior la localización debe realizarse por otros medios, empleando sensores internos: odometría [6] (giro de las ruedas) y sensores inerciales (giróscopos y acelerómetros), y sensores que realizan una medida externa al vehículo para corregir los errores acumulativos provocados por los sensores internos. Como sensores internos se suelen utilizar LIDAR 
o Sonar, y cámaras. Sin embargo, el uso de estos sensores en entornos interiores está muy desarrollado en los entornos interiores más habituales, como el interior de edificios donde existe una estructura geométrica regular, compuesta por paredes, puertas, columnas, etc., que se pueden identificar y cuya información geométrica es fácilmente parametrizable para ser usado por técnicas de localización o incluso para localización y creación de mapas (SLAM) [15]. Además, en este tipo de entorno interiores el suelo suele ser liso y estable, lo que no solo facilita el movimiento del vehículo, sino su propia localización.

En un entorno como en el que debe trabajar el vehículo autónomo no existe una estructura regular que pueda emplearse para resolver los problemas anteriormente mencionados, tanto la localización como incluso la creación de mapas del entorno. Por tanto, supone un reto en el seno del presente proyecto, que puede beneficiarse de la modificación del entorno introduciendo tags o elementos visualmente distinguibles en las zonas de inspección. De esta forma, con estos elementos se consiguen resolver dos cuestiones, la identificación de las zonas de inspección y la localización del vehículo en el entorno. Esto último permitirá desarrollar técnicas de localización, o incluso de SLAM para obtener mapas precisos del entorno, adaptadas a la situación especial del presente proyecto.

Resolver el problema de localización es esencial para cualquier vehículo autónomo, para poder desempeñar de forma precisa el resto de las tareas necesarias, como la planificación y el control de movimientos. En primer lugar, la planificación de las tareas de inspección se puede llevar a cabo empleando técnicas de búsquedas en grafos [12], ampliamente conocidas y que proporcionan muy buenos resultados. Estas técnicas se basan en encontrar los mejores caminos entre las zonas, en este caso de inspección, que se deben visitar minimizando algunos factores como la distancia, la energía necesaria para realizar los movimientos, la maniobrabilidad del camino que une dichas zonas o cualquier otro criterio que permita establecer un coste. Este tipo de técnicas tendrán que estar adaptadas a superar problemas en función de la dimensión del entorno y las zonas de inspección.

Una vez resuelto el problema de planificación del camino a realizar, es necesario enviar al vehículo la serie temporal de acciones que lleven desde la posición actual al destino. Este problema se suele resolver con técnicas de evitar obstáculos para realizar el movimiento de forma reactiva y garantizar un movimiento con seguridad en entornos dinámicos. En otras situaciones, donde el movimiento está fuertemente condicionado por las condiciones del entorno, se utilizan técnicas de seguimiento de trayectorias [1]. Este tipo de técnicas se utilizan en situaciones como la conducción autónoma para la circulación en un carril de la carretera, el comportamiento de seguimiento de pasillos en robot de limpieza, etc. En el caso del movimiento en entorno agrícolas, el camino también está establecido por la estructura regular del cultivo, por lo que se pueden definir trayectorias que atraviesen estos caminos prefijados y, empleando las técnicas de evitar obstáculos, el vehículo se mantenga a una distancia adecuada de las plantas y reaccionar ante obstáculos imprevistos. El trazado de estas trayectorias se puede realizar empleando curvas de Brezier o Splines, asegurando que las características cinemáticas y dinámicas del vehículo permiten realizar los giros y movimientos necesarios para ejecutar dichas trayectorias. Estos movimientos son complicados debido a las características del terreno, un suelo inestable (compuesto por tierra y piedras) en el que además pueden existir canalizaciones de instalaciones u otros obstáculos, como suele ocurrir en los invernaderos.

\section{Técnicas de visión por computador e inteligencia artificial}

Las técnicas de visión por computador comprenden mecanismos que permiten adquirir, procesar y analizar imágenes tomadas del mundo real. Tradicionalmente, los sistemas de visión por computador han sido utilizados para la extracción de características de las imágenes que permitirían evaluar o clasificar diferentes aspectos de las mismas, a veces apoyados en técnicas de Machine Learning para dichos objetivos. Pese a que los sistemas de visión tradicionales ofrecen buenos resultados desde un punto de vista de rendimiento, su potencial es menor a la hora de extraer información y patrones complejos. Hoy en día la combinación de técnicas de visión por computador, junto con técnicas de inteligencia artificial como Deep Learning, y en conjunción con arquitecturas multi-nivel que permiten mejorar su rendimiento, ofrecen un nuevo paradigma que permitirá resolver problemas complejos de forma eficiente.

El desarrollo de sistemas de reconocimiento de patrones o de aprendizaje automático ha requerido durante numerosos años de un gran trabajo de ingeniería y una considerable experiencia en el dominio, especialmente en aquellos considerados como complejos, para poder diseñar un extractor de características que transformara datos en bruto en una representación interna adecuada para que 

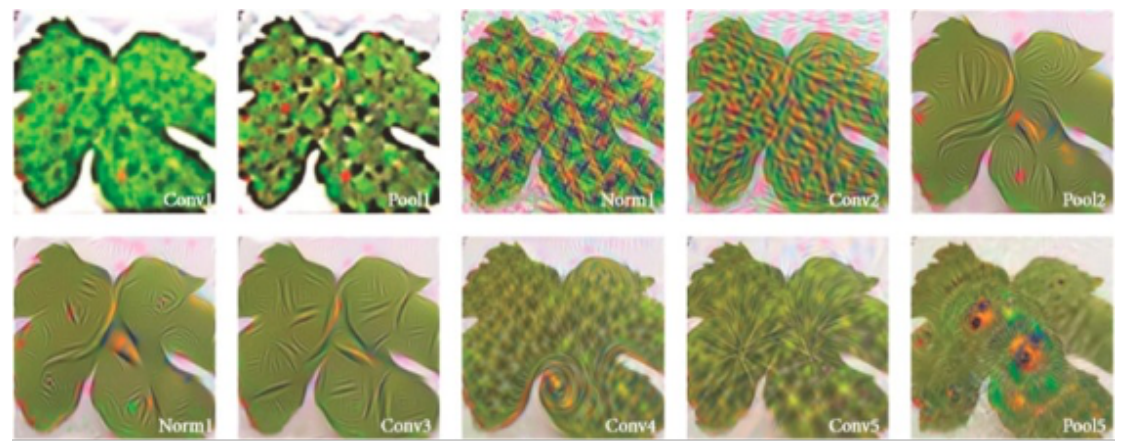

Figura 4: Visualización de las imágenes de las capas de salida después de cada paso de procesamiento de una red convolucional para la identificación de enfermedades de plantas a partir de imágenes de hojas. Fuente: [16]

los sistemas de predicción puedan realizar una correcta clasificación. Deep learning [11] está basado en técnicas de aprendizaje de representación que permiten descubrir de forma automática las representaciones necesarias para la detección o clasificación de patrones, reduciendo así el esfuerzo humano en el diseño de características. Deep learning ofrece modelos computacionales que se componen de múltiples capas de representación o procesamiento no lineales, obtenidas a partir de representaciones simples, que son utilizadas para aprender representaciones de datos con múltiples niveles de abstracción. Por tanto, una sola capa neuronal puede aprender una nueva representación simple a través de datos de entrada, y de forma conjunta, las técnicas de deep learning pueden aprender representaciones complejas. Las técnicas de deep learning han mejorado notablemente el estado del arte en reconocimiento de voz, detección de objetos y muchos otros dominios, como el descubrimiento de fármacos.

En el contexto de la agricultura [10], las técnicas de deep learning se han utilizado ampliamente para la detección de plagas. Cabe destacar las redes neuronales convolucionales, que permiten aprender características abstractas en el procesamiento de imágenes a partir de la combinación de múltiples capas convolucionales. Estas permiten detectar y/o predecir a través de imágenes no intrusivas de plantas. Las capas convolucionales actúan como extractores de características de imágenes, cuya dimensionalidad es luego reducida por las capas agrupadas. Las capas convolucionales codifican múltiples características de nivel inferior en características más discriminatorias, de una manera sensible al contexto. Un ejemplo se muestra en la Figura 4, para la detección de enfermedades en hojas. Se puede observar que después de cada paso de procesamiento, los elementos particulares de la imagen que revelan la indicación de una enfermedad se vuel- ven más evidentes, especialmente en el paso final (Pool5). Estas técnicas también han sido aplicadas con éxito para la detección de plagas como se demuestra en diversos trabajos [13], [3] y enfermedades de plantas [16]. Además de los autoencoders [18], que permiten reconstruir patrones a partir de muy escasa información de entrada, por lo que es posible detectar plagas en plantaciones hortofrutícolas con pequeños patrones. Otros enfoques, aunque en menor medida, también utilizan Differential Recurrent Neural Networks (DRNN), Long Short-Term Memory (LSTM), y deep belief networks (DBN). A pesar de los avances en este sector, actualmente existe una carencia de soluciones e implementaciones para la detección de los individuos utilizados para el control biológico, al igual que ocurre para la detección de plagas, que junto con la detección de plagas pueden ofrecer una completa visión de la biodiversidad presente en los invernadores para la toma de decisiones de los agricultores. Además, este sector necesita nuevos y completos datasets que sirvan de base a la comunidad científica y e industrial para mejorar y crear nuevas soluciones en este sector.

\section{Sistema de ayuda a la decisión: Integración de tecnologías para el control biológico y de plagas}

Como resultado del análisis previo, se presenta un sistema de ayuda a la decisión para la monitorización continua tanto de plagas que afectan a los cultivos hortofrutícolas en agricultura intensiva de invernadero, como de la lucha biológica, usado en este tipo de cultivos para combatir las plagas de una forma sostenible con el medio ambiente. Para ello, se pretende la sinergia e integración de tecnologías punteras e innovadores presentadas: 1) un vehículo de inspección autónomo que recorrerá los pasillos de los invernaderos para su continuo análisis y evaluación de la biodiversi- 


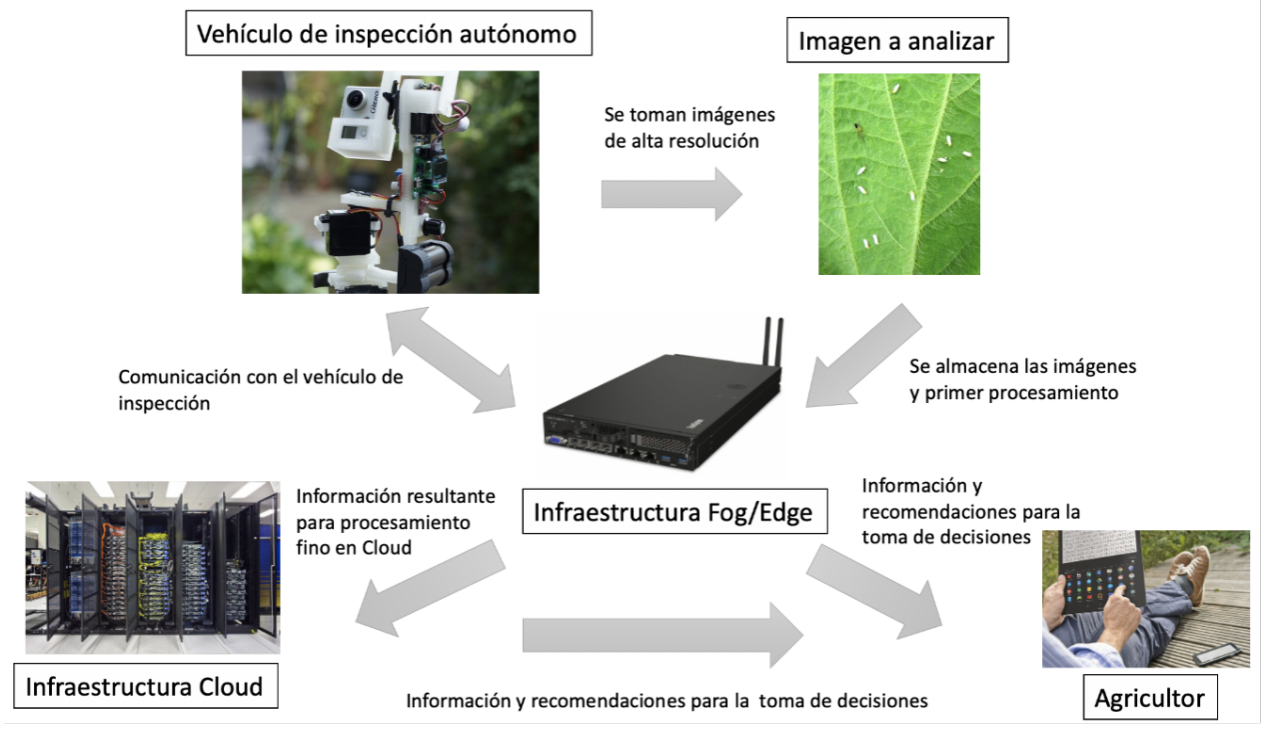

Figura 5: Visión general de la arquitectura del sistema de ayuda a la decisión para el control biológico y de plagas

dad existente; 2) técnicas de visión por computador que analizarán en tiempo real el estado de los cultivos y los depredadores y plagas existentes; 3) y una arquitectura multinivel para optimizar los tiempo de latencia y ejecución de las técnicas de visión por computador y poder actuar en la menor brevedad posible ante cualquier situación anómala o brote. Esta integración ofrecerá un nuevo sistema de monitorización continua que permitirá a los agricultores de una forma cómoda y accesible a través de sus móviles y tabletas comprobar el estado actual de sus plantaciones, ofreciendo una solución para la ayuda en la toma de decisiones en el proceso de gestión de plagas.

El vehículo de inspección autónomo no intrusivo se desplazará por los pasillos de los invernaderos para monitorizar de forma continua el estado de los cultivos con respecto a las plagas existentes y el control biológico. Aparte de la gestión de trayectorias que serán necesarias para que el vehículo se desplace de la forma adecuada y esperada por los invernaderos, y su correcto diseño para que el vehículo no tenga problema durante su trayectoria, el vehículo deberá de contar con un sistema móvil de visión que permita capturar imágenes de alta resolución para la detección de las especies presentes en los invernaderos y en posiciones de difícil acceso como el envés de las hojas.

Las técnicas de visión por computador permitirán analizar en tiempo real las imágenes capturadas por el vehículo de inspección para identificar la biodiversidad existente en los invernaderos y poder predecir el futuro estado de los mismos. Estas técnicas se desarrollarán teniendo en cuenta que pueden ser desplegadas de forma dis- tribuida en infraestructuras multinivel para la optimización de su ejecución y tiempos de respuesta.

Por último, nuevas arquitecturas multinivel permitirán desbloquear la flexibilidad de las anteriores técnicas de análisis en invernaderos en paradigmas como edge y fog computing, y habilitar su gestión, despliegue y monitorización para optimizar la respuesta del sistema. Las técnicas de análisis serán distribuidas de forma eficiente a través de estas capas, en lo que es conocido como el Cloud-to-things continuum, con el objetivo de reducir la latencia del sistema y actuar lo antes posible ante cualquier plaga o escasez de control biológico. Estas arquitecturas requerirán de nuevas capas de comunicación que puedan analizar la infraestructura existente y puedan conectar el conjunto de técnicas y procesamiento de una forma ininterrumpida y segura.

Para la consecución del sistema de ayuda a la decisión será necesaria la perfecta integración de los anteriores desafíos con el fin de que el vehículo de inspección se desplace por la trayectoria indicada por los invernaderos y se pueda analizar en tiempo real el estado de los cultivos a partir de técnicas de visión distribuidas en las arquitecturas multinivel. Todo ello con el fin de ofrecer a los agricultores un sistema de ayuda a la decisión y con el que podrán visualizar el estado de sus plantaciones (tanto de plagas como de control biológico) de una forma cómoda y accesible a través de sus smartphones o tablets. 


\section{Conclusiones y Trabajos futuros}

El camino hacia la producción sostenible en la agricultura necesita de la mejora en la gestión de plagas y la adopción de mecanismos como el control biológico de plagas que reduce drásticamente el uso de pesticidas. Aunque existen diversas soluciones que abordan ciertos aspectos en este sentido, como la detección de enfermedades y brotes de plagas en invernaderos, actualmente existe una carencia de soluciones que aborden el tan importante control biológico para lograr un perfecto equilibrio entre depredadores y parásitos y favorecer el óptimo desarrollo de los cultivos hortofrutícolas. El control biológico y de plagas necesitan una monitorización continua por parte de los agricultores y las nuevas tecnologías pueden ayudar, no solo a facilitar su labor sino a poder anticiparse a la aparición de plagas y a controlar, de forma más precisa, la lucha biológica. En este trabajo se hace una revisión de tres tecnologías habilitadoras para promover un sistema autónomo para la monitorización continua de plagas y control biológico en invernaderos. Estas líneas se engloban en: arquitecturas multinivel para la optimización de latencia; vehículos de inspección autónomos; y técnicas de inteligencia artificial y visión por computador. Por último, se presenta una integración de las mismas en un sistema que sirva de soporte en la toma de decisiones para los agricultores.

Como trabajo futuro se espera que el sistema propuesto sea implementado y validado en una explotación hortofrutícola en invernaderos, y en particular, en plantaciones de Pimiento California, que servirán como primera experiencia para poder extrapolar los resultados a otras frutas y hortalizas dónde se requiera de un control de plagas y control biológico.

\section{Agradecimientos}

Este trabajo está financiado por los proyectos RT2018-099777-B-100 ("rFOG: Improving latency and reliability of offloaded computation to the FOG for critical services"), PY20_00788 ("IntegraDos: Providing Real-Time Services for the Internet of Things through Cloud Sensor Integration") y UMA18FEDERJA-215 ("Advanced Monitoring System based on Deep Learning Services in Fog"). Colaboración con el Grupo de Investigación en Robótica y Mecatrónica de la Universidad de Málaga.

\section{English summary}

Review of enabling technologies for pest and biological control in the horticultural sector

\section{Abstract}

Continuous monitoring of pests affecting vegetable crops in intensive greenhouse agriculture as well as biological control, used in this type of crops to combat pests in an environmentally sustainable way, are essential in this sector. Currently, this control is done manually in most cases (not always without difficulty), which requires farmers to continuously monitor to detect, in the shortest possible time, any outbreak affecting their crops. This paper presents a review of enabling technologies to allow continuous, sustainable and autonomous monitoring in this strategic sector in Spain. These technologies are integrated in a decision support system for pest control and biological control.

Keywords: biological control; pest control; horticultural sector; multilevel architectures; autonomous inspection vehicle; artificial intelligence

\section{Referencias}

[1] M. Abdelbar and R. M. Buehrer. Indoor localization through trajectory tracking using neural networks. In MILCOM 2017-2017 IEEE Military Communications Conference (MILCOM), pages 519-524. IEEE, 2017.

[2] N. Ahmed, D. De, and I. Hussain. Internet of things (iot) for smart precision agriculture and farming in rural areas. IEEE Internet of Things Journal, 5(6):4890-4899, 2018.

[3] X. Cheng, Y. Zhang, Y. Chen, Y. Wu, and Y. Yue. Pest identification via deep residual learning in complex background. Computers and Electronics in Agriculture, 141:351-356, 2017.

[4] J. de Andalucia. Gestión integrada de plagas. Disponible online: https://bit.ly/ 362Cvid. (accedido el 30 de Junio de 2021).

[5] M. Díaz, C. Martín, and B. Rubio. State-ofthe-art, challenges, and open issues in the integration of internet of things and cloud computing. Journal of Network and Computer applications, 67:99-117, 2016. 
[6] J. Engel, V. Koltun, and D. Cremers. Direct sparse odometry. IEEE transactions on pattern analysis and machine intelligence, 40(3):611-625, 2017.

[7] T. N. Gia, L. Qingqing, J. P. Queralta, Z. Zou, H. Tenhunen, and T. Westerlund. Edge ai in smart farming iot: Cnns at the edge and fog computing with lora. In 2019 IEEE AFRICON, September 25-27, Accra, Ghana, pages 1-6. IEEE, 2019.

[8] E. Guardo, A. Di Stefano, A. La Corte, M. Sapienza, and M. Scatà. A fog computingbased iot framework for precision agriculture. Journal of Internet Technology, 19(5):14011411, 2018.

[9] C. Hu, W. Bao, D. Wang, and F. Liu. Dynamic adaptive dnn surgery for inference acceleration on the edge. In IEEE INFOCOM 2019-IEEE Conference on Computer Communications, pages 1423-1431. IEEE, 2019.

[10] A. Kamilaris and F. X. Prenafeta-Boldú. Deep learning in agriculture: A survey. Computers and electronics in agriculture, 147:7090, 2018.

[11] Y. LeCun, Y. Bengio, and G. Hinton. Deep learning. nature, 521(7553):436-444, 2015.

[12] S. Li, Z. Zheng, W. Chen, Z. Zheng, and J. Wang. Latency-aware task assignment and scheduling in collaborative cloud robotic systems. In 2018 IEEE 11th International Conference on Cloud Computing (CLOUD), pages 65-72. IEEE, 2018.

[13] L. Liu, R. Wang, C. Xie, P. Yang, F. Wang, S. Sudirman, and W. Liu. Pestnet: An endto-end deep learning approach for large-scale multi-class pest detection and classification. IEEE Access, 7:45301-45312, 2019.

[14] A. W. Malik, A. U. Rahman, T. Qayyum, and S. D. Ravana. Leveraging fog computing for sustainable smart farming using distributed simulation. IEEE Internet of Things Journal, $7(4): 3300-3309,2020$.

[15] J. M. Santos, D. Portugal, and R. P. Rocha. An evaluation of $2 \mathrm{~d}$ slam techniques available in robot operating system. In 2013 IEEE International Symposium on Safety, Security, and Rescue Robotics (SSRR), pages 1-6. IEEE, 2013.

[16] S. Sladojevic, M. Arsenovic, A. Anderla, D. Culibrk, and D. Stefanovic. Deep neural networks based recognition of plant diseases by leaf image classification. Computational intelligence and neuroscience, 2016, 2016.
[17] S. Teerapittayanon, B. McDanel, and H.T. Kung. Branchynet: Fast inference via early exiting from deep neural networks. In 2016 23rd International Conference on Pattern Recognition (ICPR), pages 2464-2469. IEEE, 2016.

[18] K. Trang, L. TonThat, and N. G. M. Thao. Plant leaf disease identification by deep convolutional autoencoder as a feature extraction approach. In 2020 17th International Conference on Electrical Engineering/Electronics, Computer, Telecommunications and Information Technology (ECTICON), pages 522-526. IEEE, 2020.

[19] R. Vincent, B. Limketkai, and M. Eriksen. Comparison of indoor robot localization techniques in the absence of gps. In Detection and Sensing of Mines, Explosive Objects, and Obscured Targets $X V$, volume 7664, page 76641Z. International Society for Optics and Photonics, 2010.

[20] M. A. Zamora-Izquierdo, J. Santa, J. A. Martínez, V. Martínez, and A. F. Skarmeta. Smart farming iot platform based on edge and cloud computing. Biosystems engineering, 177:4-17, 2019.

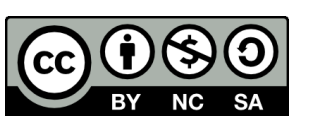
(C) 2021 by the authors. Submitted for possible open access publication under the terms and conditions of the Creative Commons Attribution CC BY-NC-SA 4.0 license (https://creativecommons.org/licenses/by-ncsa/4.0/deed.es). 